\title{
THE VARIATION OF LATITUDE
}

\author{
HAROLD JEFFREYS
}

St. John's College, Cambridge, England

\begin{abstract}
The 14-monthly variation of the Earth's axis is a free oscillation, and like others it is damped, and must be maintained by some irregular disturbance. In addition there are complications from the annual variation and observational error. The method adopted is first to analyse the data from 1899 to 1967 over 7-yr intervals, to eliminate the free period as well as possible and leave a mean value of the annual terms. The mean displacements fluctuated considerably. With a unit $0.001^{\prime \prime} l(=x)$ ranged from -15 to $+53, m(=-y)$ from the start to 1947 from -41 to +3 , in both cases quite irregularly, but $m$ reached -164 in 1955-61 and has reached -225 in 1962-8. The apparent standard errors are mostly about 6 . This behaviour is inconsistent with any hypothesis of a uniform rate of drift in either the pole or the observatories. A smooth formula for interpolating the means was adopted; this and the mean annual terms were subtracted, and the remainder was analysed for harmonic terms of 14month period. Drift of phase gave a correction to this trial period. A model on Yule's lines was used to estimate the parameters in a disturbed system. The remaining observational error over 14-month intervals turned out to be very small. The final period was $433 \cdot 15 \pm 2 \cdot 23$ mean solar days. The damping factor $e^{-k}$ in 14 months gave $k=0.05 \pm 0.034$, suggesting a time of relaxation between 14 and $73 \mathrm{yr}$, with a most probable value of $23 \mathrm{yr}$.
\end{abstract}

The 14-monthly variation, otherwise known as the Eulerian nutation and the free nutation, is a free vibration, and like most maintained free vibrations it is subject to damping. It must therefore be maintained by some irregular disturbance. For instance, the note of a violin string is maintained by irregularities in the friction of the bow, that of a wind instrument by turbulence at the orifice. In a motion of this sort ordinary harmonic analysis is useless, since disturbances separated by many periods are independent and may give rise to a spurious apparent period or to failure to detect a real one. Numerous illustrations are given in a work by M. G. Kendall. A valuable model is given by G. U. Yule. Displacements at successive instants are supposed to be connected by a difference equation of the form

$$
u_{r}-a_{1} u_{r-1}+a_{2} u_{r-2}= \pm \tau \text {. }
$$

Disturbances in different intervals are supposed independent. If $\tau$ was 0 we could assume $u_{r}$ proportional to $e^{-\alpha r}$, and get

$$
e^{-2 \alpha}-a_{1} e^{-\alpha}+a_{2}=0 \text {. }
$$

For damped harmonic motions the roots are complex; put $e^{-\alpha}=k e^{i v}$, and we find

$$
a_{1}=2 k \cos v, \quad a_{2}=k^{2} .
$$

The likelihood for $n$ observations is of the form

$$
\left(\frac{1}{2 \pi \sigma^{2}}\right)^{(n-2) / 2} \exp \left(-S \frac{\left(u_{r}-a_{1} u_{r-1}+a_{2} u_{r-2}\right)^{2}}{2 \tau^{2}}\right)
$$


and the sum in a long series approximates to

$$
n\left\{\left(1+a_{1}^{2}+a_{2}^{2}\right) \overline{u_{r}^{2}}-2\left(a_{1}+a_{1} a_{2}\right) \overline{u_{r} u_{r-1}}+2 a_{2} \overline{\left(u_{r} u_{r-2}\right)}\right\} .
$$

This is made a minimum by suitable choice of $a_{1}, a_{2}$ and uncertainties can be found as usual. All the information is summed up in the three covariances $\overline{u_{r}^{2}}, \overline{u_{r} u_{r-1}}, \overline{u_{r} u_{r-2}}$.

Unfortunately there is a complication because the observed values usually have an observational error; this tends to increase $\overline{u_{r}^{2}}$ without affecting the other two, and there is no simple way of estimating it. What we can do is to work out the expectation of some other $\overline{u_{r} u_{r-p}}(p>2)$ and try to extrapolate back to $p=0$. But these are correlated and the process is difficult.

For the free nutation, with right-handed axes, and including a damping term, the equations of motion are

$$
l+k l+\gamma m=0, \quad \dot{m}+k m-\gamma l=0 .
$$

These are of the first order, so that in this respect the problem is a little simpler than Yule's.

If the initial values are $l_{0}, m_{0}$ the solution is

$$
\begin{aligned}
l & =l_{0} e^{-k t} \cos \gamma t-m_{0} e^{-k t} \sin \gamma t \\
m & =l_{0} e^{-k t} \sin \gamma t+m_{0} e^{-k t} \cos \gamma t .
\end{aligned}
$$

If the observations are at intervals of time $\tau$ we put

$$
\alpha=e^{-k \tau} \cos \gamma \tau, \quad \beta=e^{-k \tau} \sin \gamma \tau .
$$

If there is a disturbance of each coordinate $\pm \sigma$ in any interval, we get the joint probability

$$
\begin{aligned}
P\left(\mathrm{~d} l_{1} \mathrm{~d} l_{2} \ldots \mathrm{d} l_{n} \mathrm{~d} m_{1} \mathrm{~d} m_{2} \ldots \mathrm{d} m_{n} \mid l_{0} m_{0} \alpha \beta H\right)= \\
=\left(\begin{array}{c}
1 \\
2 \pi \sigma^{2}
\end{array}\right)^{n} \exp \left[-\begin{array}{c}
1 \\
2 \sigma^{2}
\end{array}\left\{S l_{r}^{2}+S m_{r}^{2}+\left(\alpha^{2}+\beta^{2}\right) S\left(l_{r-1}^{2}+S m_{r-1}^{2}\right)-\right.\right. \\
\left.\left.-2 \alpha S\left(l_{r} l_{r-1}+m_{r} m_{r-1}\right)+2 \beta S\left(l_{r} m_{r-1}-m_{r} l_{r-1}\right)\right\}\right] \mathrm{d} l_{1} \ldots \mathrm{d} l_{n} \mathrm{~d} m_{1} \ldots \mathrm{d} m_{n} .
\end{aligned}
$$

Thus the relevant functions of the observations are the means of

$$
l_{r}^{2}+m_{r}^{2}, \quad l_{r} l_{r-1}+m_{r} m_{r-1} \text { and } l_{r} m_{r-1}-m_{r} l_{r-1} .
$$

(Some attempts have treated $l$ and $m$ separately, especially by harmonic analysis, thus losing a great deal of information).

If this was all, the matter would be straightforward. But the first complication is that besides the free motion there is a forced motion of period $1 \mathrm{yr}$, probably due to meteorological causes. We must first try to eliminate it. The free period is close to 14 months, and the most effective way is to consider means over intervals of $7 \mathrm{yr}$. Using 
the ILS data from 1899 December to 1967 July I get mean values of the annual terms

$$
\begin{gathered}
l=-66 \cos L-62 \sin L \\
m=60 \cos L-45 \sin L .
\end{gathered}
$$

The unit is 0.001 "; the phase $L$ is measured from January 16 . The fit given by harmonic analysis of the separate 7-yr intervals for a form $c+a \cos L+b \sin L$ indicates standard errors not more than 6 for $c$ and 9 for $a$ and $b$. But the actual differences for different intervals far exceed this: $a$ (for $l$ ) ranges from -26 to $-102 ; b$ from -39 to -80 , $a^{\prime}$ (accents for $m$ ) from -7 to $+94, b^{\prime}$ from -70 to -10 . The queerest thing howeve is the behaviour of $c$. For $l$ it ranges from -15 to +53 ; for $m$ up to 1947 it ranged from -41 to +3 , and since then it increased to -164 . (Analysis for the next complete 7 -yr period gave $-225 \pm 3$ ). For $l$ throughout and $m$ up to 1947 there is no regular trend; but the fluctuations far exceed the apparent uncertainty, and are hopelessly inconsistent with any hypothesis of a uniform rate of drift in either the pole or the observatories.

A rough cubic interpolation formula was used for $c$ and $c^{\prime}$, designed to fit the means over the intervals and make the first derivative continuous. This and the mean values of the annual terms were then subtracted from all the data. The remainder should contain the 14-monthly variation, the irregular disturbance, and the observational error.

In my paper of 1940 I replaced the data by means over intervals of $0.3 \mathrm{yr}$, mainly to reduce arithmetic. Walker and Young pointed out that the data had been smoothed, which would upset all the covariances. They recovered the original monthly values and argued that the shortest possible intervals should be used, namely 1 and 2 months, the latter to give another equation to estimate the observational error. I was not sure of the generality of their argument. More seriously, since weather certainly fluctuates over seasons, I doubted the hypothesis that disturbances in monthly intervals were independent.

It seemed that the most hopeful way was to make harmonic analyses for 14-monthly intervals; disturbances in these would have more chance of being independent, and the cosine and sine terms in $l$ and $m$ would be related just as before. If the period was a little wrong this would be detected in the analysis - the new $2 \pi \gamma$ being the phase shift in 14 months reduced by $2 \pi$. It was also possible to use analyses covering $2,4,8$ and 14 -monthly periods. It turned out that analysis combining 14-monthly periods gave the most accurate determinations.

However this still does not deal with the effect of observational error. It does not affect the estimate of the period but it can inflate $S\left(l^{2}+m^{2}\right)$ and hence overestimate the damping. But expectations come to the rescue after all. Comparing $S\left(a^{2}+b^{2}\right)$ for the different lengths used led to a slightly negative value of $\sigma^{2}$, where $\sigma^{\prime}$ is the observational standard error of $a$ datum coefficient. This of course was impossible. A check was found from the apparent uncertainties of the harmonic coefficients in the 14month intervals, which were about 9 units and would include both observational error and short-range parts of the disturbance. This led to $0 \leqslant \sigma^{\prime 2}<200$. 
The resulting period is $1.1859 \pm 0.0061 \mathrm{yr}$

$$
\begin{aligned}
& =433.15 \pm 2.23 \text { mean solar days } \\
& =434.34 \pm 2.23 \text { sidereal days. }
\end{aligned}
$$

If $e^{-k}$ is the damping factor in 14 months, $k$ as corrected for $\sigma^{\prime}$ is $0.05 \pm 0.034$; the probability of the time of relaxation is far from normally distributed, but limits corresponding to the standard error are 14 to $73 \mathrm{yr}$. A consistent result has been found by Arato. We should certainly like a closer estimate of $k$, but it looks as if $200 \mathrm{yr}$ observations will be needed to make a really substantial improvement. But what we have got is enough to be useful; chiefly because we have got an estimate of damping for the longest periodic motion of the Earth.

I have not dwelt here on causes. The fluctuation of the means over 7-yr intervals is quite unexplained; the usual ideas of constant rates of drift are quite unsatisfactory. The greater part of the annual motion is definitely due to seasonal variation of the distribution of air over the Earth. But this by itself would be about six weeks wrong in phase. I suggested annual variation of snowfall as giving an important correction; Munk and MacDonald suggest annual variation in ground water. I have removed the mean values of the annual motion from the data, and it may be asked why I did not use the 7-yr values and interpolate as for the means. The reason is that whatever produces a slow fluctuation of the annual motion is precisely what is needed to maintain the free motion.

\section{Reference}

Jeffreys, H.: 1968, Monthly Notices Roy. Astron. Soc. 141, 255.

\section{DISCUSSION}

P. Melchior: You have used the original ILS data for your analysis. Has the drift of the mean pole an effect on the determination of the damping factor in your method of analysis?

$H$. Jeffreys: The preliminary analysis of the coordinates was meant to determine any systematic drift, and more detailed corrections to it were found in the analysis of 14 month periods. So I hope that it has been cleared out. 